\title{
REPRESENTAÇÕES SOCIAIS SOBRE A ALIMENTAÇÃO POR SONDA OBTIDAS DE PACIENTES ADULTOS HOSPITALIZADOS
}

Jaqueline Almeida Guimarães Barbosa ${ }^{1}$ Maria Imaculada de Fátima Freitas ${ }^{2}$

Barbosa JAG, Freitas MIF. Representações sociais sobre a alimentação por sonda obtidas de pacientes adultos hospitalizados. Rev Latino-am Enfermagem 2005 março-abril; 13(2):235-42.

Identificar e compreender as representações de pacientes sobre alimentação por sonda. Entrevista aberta com 16 pacientes em uso de sondas para alimentação. A análise das entrevistas foi fundamentada na análise de discurso. O referencial teórico adotado foi a Teoria das Representações Sociais. Identificou-se a existência de uma trajetória, na qual as representações vão sendo reorganizadas e reconstruídas pela experiência, para tornar tolerável esse tratamento, começando quando o paciente se depara com a necessidade do uso, passando pela experiência do uso e, finalmente, tendo que se decidir pela continuidade ou não do uso no ambiente domiciliar. Dentre as representações encontradas, existem algumas que contribuem para a adesão e outras para a rejeição a esse tratamento. Conhecendo as idéias e crenças positivas e negativas sobre esse tratamento, torna-se possível atuar de forma mais direcionada a fim de aumentar a adesão ao tratamento e satisfação com ele.

DESCRITORES: nutrição enteral; antropologia cultural; sociologia

\section{SOCIAL REPRESENTATIONS ASSOCIATED WITH TUBE FEEDING AMONG ADULT HOSPITALIZED PATIENTS}

This study aimed to identify and understand adult patients' representations on tube feeding. Data were collected through interviews with sixteen patients who were using feeding tubes, which were analyzed through discourse analysis and Social Representation Theory. We identified a sequence in which representations are reorganized and redefined through experience, in order to turn this treatment tolerable. This sequence begins when patients face the need of a feeding tube, followed by the experience of its use and finally, when deciding about continuing its use or not, when going home. Some representations contribute to treatment adherence and others to its rejection. Getting to know positive and negative ideas about this treatment makes it possible to approach these patients in a more direct way, with a view to better adherence and satisfaction.

DESCRIPTORS: enteral nutrition; anthropology, cultural; sociology

\section{REPRESENTACIONES SOCIALES ASOCIADAS CON LA NUTRICIÓN POR SONDAS EN PACIENTES HOSPITALIZADOS}

La finalidad fue identificar y comprender las representaciones asociadas con la nutrición por sondas en pacientes hospitalizados. Fueron realizadas entrevistas con 16 pacientes en uso de sondas para nutrición enteral. El análisis de las entrevistas tuvo como base el análisis del discurso y la Teoría de Representación Social. Fue identificada una secuencia en que las representaciones son reorganizadas y reconstruidas por la experiencia, para hacer el tratamiento tolerable. Esta secuencia se inicia cuando el paciente tiene que enfrentar la necesidad del uso de la sonda, seguida por la experiencia de su uso y, finalmente, cuando tiene que decidir sobre la permanencia o no de la sonda cuando se va al hogar. Entre las representaciones encontradas existen algunas que contribuyen para la adhesión y otras para su rechazo. Con base en las representaciones identificadas, es posible actuar de manera mas dirigida para aumentar la adhesión y satisfacción con el tratamiento.

DESCRIPTORES: nutrición enteral; antropología cultural; sociología

\footnotetext{
${ }^{1}$ Mestre em Enfermagem, Especialista em Terapia Nutricional Enteral e Parenteral, Membro da Equipe Multidisciplinar de Terapia Nurticional do Hospital Luxemburgo, e-mail: jaqueline@taskmail.com.br; ${ }^{2}$ Doutor em Ciências da Educação, Coordenadora do Colegiado de Pós-Graduação da Escola de Enfermagem da Universidade Federal de Minas Gerais, e-mail: peninha@enf.ufmg.br
} 


\section{INTRODUÇÃO}

A alimentação é condição essencial para a sobrevivência humana. No entanto, o índice de desnutrição de pacientes hospitalizados é alarmante, em torno de $48 \%{ }^{(1)}$. Pacientes hospitalizados não se alimentam suficientemente para atingir suas necessidades calóricoprotéicas devido aos mais variados fatores, como a doença de base, dor, náuseas, vômitos, ansiedade, inapetência, disfagia, depressão, incapacidade funcional, tratamentos agressivos como cirurgias, rádio e quimioterapia, e mesmo pelo ambiente hospitalar. Em decorrência disso, o estado geral e a resposta aos tratamentos ficam comprometidos, além de as complicações serem entre duas e vinte vezes maiores, quando comparados aos enfermos nutridos ${ }^{(2)}$. Diante desse cenário, a terapia nutricional tem conquistado, a cada dia, maior reconhecimento de sua importância, tanto para a recuperação do estado nutricional quanto para a sua manutenção. Quando bem indicada, melhora os resultados clínicos, reduzindo o tempo de internação e os custos com a saúde. É sabido que pacientes bem nutridos respondem melhor aos diversos tipos de tratamento. No entanto, ao alimentar-se, os indivíduos não estão satisfazendo somente a suas necessidades fisiológicas, mas também a muitas necessidades psicossociais. Além do aspecto nutritivo, a alimentação traz consigo diversas significações e implicações na vida das pessoas ${ }^{(3)}$. Logo, pode-se inferir o quão desestruturador pode ser estar impossibilitado de se alimentar por via oral e ser alimentado por vias artificiais, como por sondas. Estando em uso de nutrição enteral, que elimina o prazer do sabor e cheiro proporcionado pelos alimentos, mantendo sempre a mesma cor, aspecto e consistência, o processo nutritivo toma outras dimensões. Para esses pacientes, o momento de alimentar-se sob essas condições não corresponde mais a um momento de integração e troca de afetos, pois passa a representar tensão, angústia e discriminação, intensificados pelos sentimentos de abandono, desvalia e insegurança ligados à hospitalização ${ }^{(4-5)}$. Nessas situações ocasionadas pela ruptura de tempo e de espaço do convívio familiar, os sentimentos de não mais fazer parte do contexto social são aumentados pelo isolamento vivido nos momentos das refeições. Não é mais um momento de partilhamento e nem de prazer, mas de obrigação de se alimentar para não morrer. A nutrição enteral é percebida, na maioria das vezes, como não desejada, mas imposta, trazendo consigo uma carga de representação afetiva de desvinculação social. Ela se transforma, muitas vezes, em fator gerador de estresse para o paciente e suas famílias. Apesar de nos encontrarmos diante de um cenário de grande desenvolvimento científico e tecnológico, no tocante aos recursos disponíveis para a assistência à saúde, estes têm gerado grandes repercussões na vida dos pacientes. As alterações e restrições impostas por alguns tipos de tratamento podem levar a uma drástica alteração no estilo de vida das pessoas, e este fator não pode ser subestimado. Diante da insatisfação presenciada por parte dos pacientes mediante a necessidade do uso de sondas para se alimentar, e sabendo da importância da alimentação para a manutenção e recuperação da saúde das pessoas ${ }^{(6-7)}$, este trabalho teve como objetivo identificar e compreender as representações de pacientes adultos hospitalizados sobre a alimentação por meio de sondas. Refletir sobre as representações relacionadas à alimentação por sondas poderá contribuir para encontrar respostas que os profissionais de saúde precisam e querem conhecer, a fim de que suas condutas sejam tomadas respeitando os limites, a dignidade e os direitos dos pacientes. Acreditase que estudos dessa natureza possam apontar novas abordagens na dimensão do cuidado integral, melhorar a adesão a esse tratamento, quando esse tipo de terapia se fizer necessário, e proporcionar melhor qualidade de vida para esses pacientes.

\section{MATERIAL E MÉTODOS}

Foi utilizada como referencial teórico-metodológico a Teoria das Representações Sociais (RS). Ela enfoca questões relacionadas às idéias e crenças que interferem nas atitudes das pessoas diante da necessidade de tratamento, incluindo nutrição enteral por meio de sondas. ARS pode ser definida como uma atividade mental pela qual um indivíduo ou um grupo reconstitui o real com o qual ele é confrontado e lhe atribui uma significação específica. ARS funciona como um sistema de interpretação da realidade, o qual rege as relações dos indivíduos no seu contexto físico e social e determina os seus comportamentos ou práticas. Ela é um guia para a ação, ela orienta as ações e as relações sociais. A representação constitui um universo de opiniões e de crenças organizadas em torno de uma significação central e em relação a um objeto determinado. Esse núcleo é o elemento ou conjunto de elementos que permitem à representação social, a explicitação de sua significação e coerência. Ele gera, organiza e estabiliza a representação. Em torno desse núcleo central, agrupam-se um conjunto de informações, de atitudes e de crenças e um sistema de categorização. Há, portanto, elementos que são definidores ou necessários, chamados núcleos centrais, enquanto outros são contingentes ou acessórios, chamados de núcleos periféricos. Assim, o núcleo central funda a coerência e a 
permanência da representação, enquanto o sistema periférico admite variações inter-individuais e intra-individuais, funcionando como um dispositivo plástico e adaptativo da representação ${ }^{(8-11)}$.

A pesquisa de campo foi realizada em um hospital geral, de médio porte, filantrópico, em Belo Horizonte. Os sujeitos foram pacientes hospitalizados em uso de sondas para alimentação (sonda nasoentérica, gastrostomia ou jejunostomia). Foram entrevistados somente pacientes adultos, considerando a sua capacidade de verbalização. Os pacientes foram provenientes das diversas clínicas e internados por meio de Convênios e pelo Sistema Único de Saúde (SUS). Foram excluídos pacientes inconscientes, sedados, disfásicos e confusos. O número de participantes foi definido segundo o critério de exaustividade e saturação das informações, mediante a análise preliminar realizada no transcorrer da coleta, tendo sido realizadas 16 entrevistas. Como instrumento para a coleta das informações, foi utilizada a entrevista aberta, com questões sobre as representações dos pacientes a respeito do uso de sondas para se alimentar. As entrevistas foram gravadas e, em seguida, transcritas pela pesquisadora. Dados de identificação foram coletados utilizando-se o prontuário, o qual também continha informações sobre todo o tratamento nutricional. Para a realização das entrevistas, após o esclarecimento sobre a pesquisa, foi solicitada assinatura do Termo de Consentimento Livre e Esclarecido de cada entrevistado, aprovado pelo COEP. Foi explicitado que poderiam se recusar a participar da pesquisa a qualquer momento, sem qualquer prejuízo em seu tratamento. Para a contextualização do grupo de sujeitos participantes da pesquisa, foram coletadas informações referentes à idade, sexo, diagnóstico, nível de escolaridade, estado civil, número de filhos, diagnóstico principal, estado nutricional, tipo de relação no sistema de saúde, tempo médio de hospitalização, possibilidade de uso da via oral concomitante ao uso da sonda, presença de acompanhante, tempo de uso da sonda definitiva e qual a evolução obtida no tratamento, ou seja, se obteve alta com ou sem sonda nasoentérica, ou se teve indicação de uso da sonda definitiva.

A análise das entrevistas foi realizada em três etapas, fundamentada na análise de discurso ${ }^{(12)}$. Na primeira etapa, fez-se a transcrição das entrevistas, no decorrer da coleta de dados, com análise preliminar para encontrar os temas comuns e discordantes surgidos no conjunto das entrevistas, para haver definição da suspensão de novas entrevistas, utilizando-se dos critérios de exaustividade e saturação dos temas. Já numa segunda etapa, cada entrevista foi analisada para se definirem as unidades temáticas, caracterizadas pelos objetos da fala ${ }^{(13)}$ ou fatos relatados, com as justificativas e os sentimentos sobre eles. Em seguida, ainda nessa segunda etapa, buscou-se a definição dos núcleos centrais e dos periféricos das representações ali contidas, que recebiam uma denominação preliminar referente a cada uma. $\mathrm{Na}$ terceira etapa, na qual se analisou o conjunto das entrevistas de forma transversal, realizou-se o agrupamento das unidades temáticas referentes às representações emergidas das falas, com seus núcleos centrais e periféricos, buscando a denominação mais pertinente a elas. Finalmente, as representações encontradas foram discutidas com referências teóricas relativas a cada uma. Nessa última etapa, as diversas representações encontradas são agrupadas em torno do objeto central do discurso, determinando o núcleo central e os periféricos de cada representação, que recebem denominações que garantem a fidedignidade das informações.

\section{RESULTADOS}

As representações centrais e periféricas nos discursos de pacientes hospitalizados em uso de sonda para alimentação

Verificou-se uma trajetória no pensamento dos indivíduos ao se depararem com a experiência da alimentação por meio de sondas vivenciando-a, fazendo-os desconstruir e reconstruir novas representações de acordo com a vivência de cada um. Essa trajetória determinou a experiência do uso de sonda para alimentação em três fases: representações presentes anteriormente ao uso da sonda para se alimentar, afloradas ao se depararem com a necessidade do seu uso; representações desconstruídas e reconstruídas durante o uso; e, por fim, representações específicas dos sujeitos em uso de cada tipo de sonda, quando diante da necessidade da continuidade do seu uso no ambiente domiciliar.

As representações identificadas em cada fase decorrem da interação com as diversas situações que surgem, como do paciente consigo mesmo, com seus familiares, com a sociedade, com a equipe de saúde e com as diversidades que essa realidade possa the apresentar, como as possíveis complicações desse tratamento. 0 conjunto das representações identificadas em cada fase está colocado, de forma esquematizada, para melhor visualização, na Figura 1. Os núcleos centrais identificados estão na coluna central, agrupados de acordo com o momento em que aparecem na trajetória (coluna da esquerda). Os núcleos periféricos que sustentam cada núcleo central estão dispostos à direita de cada representação central. 


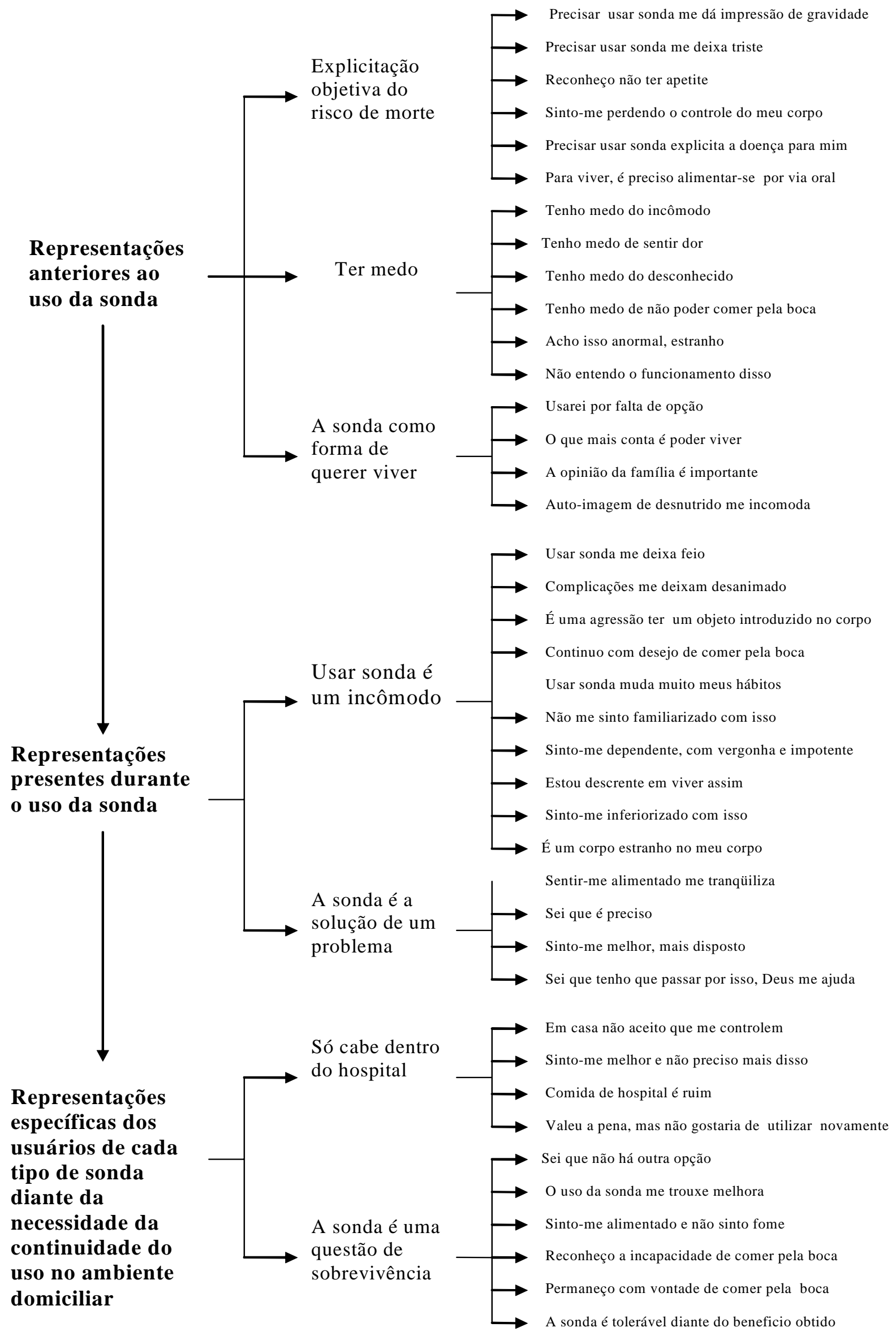


Representações presentes anteriormente ao uso da alimentação por sondas

Ao se depararem com a necessidade do uso de sondas para se alimentar, três núcleos centrais foram identificados nos discursos dos entrevistados: 'a necessidade do uso da sonda representada como aproximação da morte', 'o sentimento de medo acerca dessa terapia' e 'a aceitação do uso de sonda como forma de querer viver'.

Explicitação objetiva do risco de morte

Esse foi o primeiro núcleo central identificado ao se depararem com a necessidade da alimentação por meio de sondas: uma explicitação objetiva do risco de morte. A necessidade do uso da sonda transforma-se num indicador da própria finitude, que se apresenta como uma realidade concreta nesse primeiro. Os entrevistados demonstram pavor e dificuldade para encarar esse fato. No entanto, a indicação do uso de sonda para um paciente debilitado faz-se justamente para evitar a morte, ou seja, para preservar a vida. Parece, então, que a finalidade do uso da sonda para alimentação não é um fato inicialmente compreendido pelos pacientes. Prevalece o pavor de encontrar-se frente à morte, a despeito de esse tratamento e os cuidados oferecidos estarem à sua volta para oferecer assistência e vida.

Para esse núcleo central, foram encontrados e categorizados os seguintes núcleos periféricos, que lhe dão sustentação: 'precisar de sonda me dá impressão de gravidade'; ' precisar usar sonda me deixa triste ou deixa os outros tristes'; '0 uso de sonda explicita a doença para mim'; 'precisar usar sonda me dá impressão de perda de controle sobre meu próprio corpo'; 'alimentar-se é por via oral'; 'eu não tenho apetite'.

Tenho medo

As representações sobre o uso de sonda para se alimentar concentram o medo em suas diferentes formas, configurando-se como o segundo núcleo central identificado nas representações anteriores ao uso da sonda para se alimentar. Existe o medo de sentir dor, medo do incômodo, do desconhecido, do que soa como estranho e do que seja anormal. Esses medos são reforçados pela desinformação e conseqüente insegurança do que esteja acontecendo e do que pode vir a acontecer. Aparece, também, o medo de perder a via oral como via de alimentação e de todo o prazer que ela proporciona. Todos os núcleos periféricos desse grupo mostraram-se contributivos para a recusa a esse tratamento.

Nota-se, no entanto, uma desconstrução e reconstrução das representações, o que se verifica ao longo da experiência do uso dessa terapia. Alguns medos vão desaparecendo, na medida em que os pacientes vão obtendo maiores esclarecimentos e familiaridade com o tratamento. Os pacientes percebem que receber alimentação por meio de sonda não é tão ruim quanto imaginavam e acabam se acostumando com essa nova situação e adaptando-se a ela. Os núcleos periféricos encontrados na análise e que sustentam esse núcleo central foram categorizados em: 'medo do incômodo'; 'medo da dor'; 'medo do que seja desconhecido'; 'medo de não mais poder se alimentar pela boca'; e 'não entendo o funcionamento disso'.

A sonda como forma de querer viver

Esse foi o terceiro e último núcleo central identificado nesse primeiro momento da trajetória. Os pacientes demonstram reconhecer a necessidade de ajuda para se manterem alimentados, reafirmada, inclusive, pelas falas de seus familiares. Estando eles diante do fato de se encontrarem sem alternativas, passam, então, a aceitar essa terapia como uma forma de sobrevivência. Os núcleos periféricos que sustentam essa representação central foram: 'usarei porque não há outra opção'; 'o que mais conta é poder viver'; 'sei que, sem comida, eu morro'; 'a opinião da minha família é importante nessa decisão'; 'estar desnutrido me incomoda, reflete falta de saúde'.

Representações presentes durante o uso da alimentação por meio de sondas

Foram identificadas duas representações centrais durante a experiência do uso da sonda: 'usar sonda é um incômodo' e 'usar a sonda para se alimentar é a solução de um problema'.

Usar sonda é um incômodo

Para esse núcleo central, foram identificados os seguintes núcleos periféricos que lhe dão sustentação: 'a sonda me deixa feio'; 'cada vez que aparece uma complicação me dá mais desânimo'; 'é uma agressão ter um objeto introduzido no corpo'; ' continuo com desejo de me alimentar pela boca'; 'usar sonda para alimentação muda muito meus hábitos'; 'não me sinto familiarizado com isso'; 'sinto-me dependente, com vergonha e impotente diante da realidade'; 'estou descrente em viver assim'.

Pode-se afirmar que todos os núcleos periféricos mostraram contribuir para a rejeição a esse tratamento. Aanálise também mostra que os pacientes vivem uma forma de superação dos núcleos periféricos das representações anteriores ao uso, como os de estranheza e medo, confirmando o dinamismo existente nas representações sociais. Esse é um processo de luz e sombra, no qual, mesmo que o senso comum incorporado em cada um aponte que o uso da sonda é difícil, cria-se uma familiaridade com o objeto, deixando escondido, na sombra, aquelas representações anteriores. 
A sonda é a solução de um problema

Essa foi a segunda representação central identificada nesse momento. Os núcleos periféricos identificados que sustentam essa representação foram: 'sentir-me alimentado me tranqüiliza'; 'sei que preciso'; 'sinto-me melhor e mais disposto' e 'sei que tenho que passar por isso, Deus me ajuda'.

Nessa categoria, também percebemos reafirmadas algumas representações presentes anteriormente ao uso, como na categoria das representações que vêem a sonda como questão de sobrevivência, como forma de querer viver.

Refletindo sobre a representação central da sonda como solução do problema, fica evidenciado que, mesmo vivendo o paradoxo da idéia e da prática sobre o fato de que a sonda é um incômodo, há o reconhecimento da importância da alimentação para a saúde e manutenção do organismo vivo, sendo necessário, nesse momento, 0 uso da sonda para que ela aconteça.

As representações são, como se nota, uma construção ativa, e não um estado estático. $O$ doente vai aprendendo a viver com sua "fragilidade", e a utilizar melhor o conjunto de suas capacidades defensivas tanto físicas quanto psicológicas, sendo essas potencialidades um auxílio indispensável. Isso supõe que o paciente esteja fazendo uma reavaliação profunda da imagem que ele tem de si mesmo, quando vais cada vez mais, morrendo a "imagem ideal", a fim de se adotar uma nova, mais adaptada à nova situação. $O$ doente ao longo do tratamento vai, passando por um processo no qual está se conhecendo melhor, aprendendo a falar de suas emoções, modificando eventualmente suas condições de vida e seu meio, de modo a poder adaptar-se à sua nova vida.

Pode-se concluir, diante das representações centrais identificadas durante a experiência do uso, que "usar sonda para se alimentar é um incômodo", mas mostra-se tolerável, fazendo emergir, então, a representação central de que "usar a sonda e receber a alimentação enteral é a solução de um problema" naquele momento. Esse processo conduz, na maioria das vezes, à adesão ao tratamento.

Representações específicas dos pacientes em uso de cada tipo de sonda, diante da necessidade da continuidade do tratamento no ambiente domiciliar

Somente nesse terceiro momento, foram identificadas representações específicas e diferenciadas entre os usuários de sondas definitivas (gastro e jejuonostomias) e dos usuários de sondas temporárias (sondas nasoentéricas). As especificidades aparecem decorrentes de algumas diferenças presentes entre os dois grupos, sendo a principal delas as características dos quadros clínicos. Geralmente, os pacientes em uso de sondas definitivas apresentam quadros com piores prognósticos. Esses também se encontram mais sem alternativas ou opções de escolha quanto à possibilidade de uso da via oral quando comparados aos pacientes em uso de sondas temporárias, que são contextos sobre os quais estão conscientes.

\section{Só cabe dentro do ambiente hospitalar}

Esse foi o núcleo central identificado apenas para os pacientes em uso de sonda temporária ou sonda nasoentérica, ao se depararem com a necessidade da continuidade do uso no ambiente domiciliar. Nos discursos coletados, fica evidente que, dentro do hospital, o uso da sonda é cabível, aceitável, não se estendendo, porém, ao ambiente domiciliar. De todos os entrevistados em uso da sonda nasoentérica, com exceção do que evoluiu para a colocação da sonda "definitiva", todos os outros tiveram indicação da continuidade do uso, por mais um período, no ambiente domiciliar, mas todos a recusaram. A partir do momento em que recebem a alta hospitalar, eles afirmam serem capazes de se alimentar suficientemente pela via oral no ambiente domiciliar. Eles demonstram se sentirem resgatando a autonomia sobre a sua alimentação ao retornarem para o seu lar. Ao sentirem sinais de melhora, os pacientes tendem a relaxar e abandonar seus tratamentos, interrompendo-os muitas vezes, antes do permitido. Essa atitude está presente nos mais variados quadros de adoecimento, fundamentada na representação do processo saúde/doença que identifica as necessidades somente como necessidades sentidas, e a doença somente quando há sintomas e sinais visíveis ${ }^{(14)}$.

Os núcleos periféricos que sustentam essa representação central foram assim categorizados: 'em casa não aceito que me controlem', 'comida de hospital é ruim, em casa vou comer melhor', 'já me sinto melhor e não preciso mais disso' e 'valeu a pena, mas não gostaria de utilizar novamente'.

A sonda é uma questão de sobrevivência

Esse foi o núcleo central identificado apenas nos pacientes em uso de sondas permanentes (gastrostomias ou jejunostomias), quando diante da necessidade da continuidade do uso no ambiente domiciliar. São pacientes mais impossibilitados de uso da via oral e conscientes disso. Os núcleos periféricos que emergiram dessa análise foram: 'não há outra opção'; ' 'o uso da sonda me trouxe melhora'; 'me sinto alimentado e aliviado por não sentir fome'; 'sei que não consigo me alimentar sozinho'; 'apesar de saber que a sonda me alimenta, permaneço com vontade de comer pela boca' e 'a sonda é tolerável mediante o benefício alcançado'.

\section{DISCUSSÃO}

Dentre os resultados encontrados, identificaram-se tanto 
representações que levam à rejeição como representações que contribuem para a adesão a esse tratamento, as quais podem, no entanto, irem se modificando de acordo com o momento vivenciado.

Os achados estão em consonância com estudo no qual se aborda o significado da nutrição parenteral para o cliente no contexto hospitalar ${ }^{(19)}$. Apesar das diferenças existentes entre os dois tipos de alimentação e, principalmente, nas vias de inserção do alimento, muitos dos significados e representações encontrados foram os mesmos, mediante a semelhança do quadro vivenciado de restrição alimentar, pelo recebimento de alimentação "artificial" por vias que não a "normal" e pelas restrições e limitações impostas pelo tratamento, dentre outros. Eles coincidem, também, no que diz respeito ao desconhecimento acerca do tratamento que estão recebendo, à desconfiança da nutrição artificial como alimento, ao sofrimento e à tristeza sentidos ao precisar dessa terapia, na falta e no desejo sentidos do uso da alimentação pela via oral, no reconhecimento da melhora obtida e na manutenção do estado geral, no incômodo ocasionado pelas restrições e limitações impostas pelo tratamento, e na desilusão da perspectiva de vida. No entanto, percebe-se que a via de administração da nutrição parenteral, que é a endovenosa, é mais bem aceita que as vias de administração da nutrição enteral, que são as sondas, uma vez que as sondas mostram alterar muito a auto-imagem e auto-estima dos pacientes. Diante disso, podese inferir que a terapia nutricional enteral apresenta-se aos pacientes como mais invasiva e mais estranha, e, portanto, mais difícil de ser aceita. Fazem-se necessários, pois, maiores investimentos em sondas que ocasionem menores alterações possíveis na estética dos usuários e a preços acessíveis para a população carente.

Os resultados estão em consonância também com trabalhos realizados por diferentes autores que abordam a adesão de pacientes a tratamentos de doenças como diabetes, hipertensão, tuberculose e Aids. A literatura mostra que a adesão aos tratamentos sofre influência de fatores como o tempo de tratamento, a autopercepção do conceito de saúde, o suporte social da família e de amigos, que são fatores construtores das representações. Como fatores dificultadores, foram apontados o estresse, o processo de aceitação pelo indivíduo de sua doença, a associação feita entre o tratamento e a proximidade da morte, a negação de uma possível evolução da doença, a ocorrência de efeitos colaterais e também do custo dos cuidados médicos e a acessibilidade a eles. Os autores relatam que pacientes com depressão, sentimentos negativos, com perda de motivação e de expectativas no futuro têm menor adesão, fato este também constatado neste estudo. 0 suporte relacionado à informação do paciente acerca de sua situação e da evolução da doença foi a variável mais fortemente associada à melhor qualidade de vida ${ }^{(15-18)}$.

\section{CONSIDERAÇÕES FINAIS}

Fica evidente, mediante as representações encontradas, a importância de se agregarem aos cuidados prestados pelos profissionais de saúde às pessoas que necessitam de alimentação por meio de sondas, as subjetividades destas, admitindo-se que as verdades aceitas pelos sujeitos não são necessariamente somente as do objeto que se Ihes apresenta, mas uma visão representativa do mundo. Conhecendo as idéias e crenças positivas e negativas sobre esse tratamento, tornase possível maior compreensão das atitudes tomadas pelos pacientes, das repercussões que esse tratamento tem na vida deles e, conseqüentemente, fornece-nos subsídios para uma atuação mais direcionada, pois os profissionais buscam minimizar as representações que levam à rejeição do tratamento, sejam elas objetivas ou subjetivas, e fortalecer aquelas que levam à adesão a ele. Um fator que mostrou contribuir para a não continuidade do tratamento é decorrente do uso de materiais inespecíficos e de qualidade não comprovada, como as sondas impróprias para esse fim (sonda de Foley, dreno de Kehr) que acabam gerando maiores complicações e, conseqüentemente, desmotivando o doente e desacreditando da eficiência do serviço e da terapia. Existem, no mercado, produtos próprios e de qualidade comprovada, mas a preços considerados altos para o Sistema Único de Saúde. Portanto, o custo-benefício do uso desse material "improvisado" e de baixo custo deve ser considerado, uma vez que as complicações decorrentes disso podem ser muito mais onerosas, podendo custar vidas! Quanto aos medos e às alterações sentidas na auto-imagem e na auto-estima, quando em uso de sonda para alimentação, isso pode ser superado com diálogo e mostrando ao paciente que ele continua a ser uma pessoa íntegra, digna e de valor, independente de usar ou não uma sonda no corpo, assim como procurar adaptar o uso da terapia nutricional aos hábitos de vida do doente. No entanto, foram identificadas, também, representações negativas acerca desse tratamento as quais fazem parte, também, da história de vida e da personalidade dos indivíduos e que dificilmente se modificam, como aquelas relacionadas à sua filosofia de vida e à sua visão de mundo. Em algumas situações, percebe-se que conseguir alimentar-se sem depender de uma sonda é questão de honra para as pessoas. Além disso, o desejo e prazer da alimentação pela via oral é algo que dificilmente a terapia nutricional enteral terá como superar.

Os resultados encontrados apontam também para a necessidade de os profissionais de saúde refletirem sobre as suas próprias representações, uma vez que participam ativamente, mesmo que inconscientemente, do processo de construção das representações dos pacientes, com o propósito de minimizar a "transferência" de representações negativas e, sim, de enfatizar as positivas que propiciam a adesão a esse tratamento, ou seja, enfocando a importância da alimentação para a vida, o uso da sonda como uma alternativa viável, e a melhora obtida após o início do tratamento. Outro fator propiciador da adesão a ser aproveitado e reforçado é o apoio dos familiares e da religião.

Enfim, que os profissionais reflitam sobre sua atuação 
profissional diária, pois, na maioria das vezes, encontram-se inseridos em atividades burocráticas e técnicas que Ihes "roubam" um tempo valioso e necessário para se encontrarem com os doentes, conhecêlos e compreendê-los. Esse tempo refere-se à necessidade da permanência dos doentes à beira do leito, "lá, onde tudo se passa,

\section{REFERÊNCIAS BIBLIOGRÁFICA}

1. Waitzberg DL, Caiaffa WT, Correia MITD. Inquérito Brasileiro de Avaliação Nutricional Hospitalar (Ibranutri). Rev Bras Nutr Clin 1999; 14:123-33.

2. Correia MITD. Repercussões da desnutrição sobre a morbimortalidade e custos em pacientes hospitalizados no Brasil. [tese]. São Paulo (SP): Faculdade de Medicina/USP; 2000.

3. Cerezetti CCN. Aspectos psicológicos do paciente em Suporte Nutricional Artificial. In: Waitzberg DL. Nutrição Enteral e Parenteral na Prática Clínica. $2^{\mathrm{a}}$ ed. Rio de Janeiro (RJ): Atheneu; 1995. p.178-80. 4. Cascudo LC. História daAlimentação no Brasil. São Paulo (SP): Ed. Nacional; 1968.

5. MiyadahiraAMK. Princípios de assistência de enfermagem na nutrição enteral. Rev Paul Enfermagem 1984 abril/junho; 4(2):62-8.

6. Waitzberg DL. Nutrição Enteral e Parenteral na Prática Clínica. $3^{a}$ ed. São Paulo (SP): Atheneu; 2000.

7. Carvalho EB, Sales TRA. Avaliação Nutricional; a base da escolha terapêutica. In: Carvalho EB. Manual de Suporte Nutricional. Rio de Janeiro (RJ): Medsi; 1992. p.21-39.

8. Anadon M, Machado PB. Reflexões Teórico-Metodológicas sobre as Representações Sociais. Salvador (BA): Ed. UNEB; 2001.

9. Abric JC. Coopération, competition et représentations sociales. Cousset (FR): Delval; 1987.

10. Moscovici S. La psychanalyse: son image et son public. Paris (FR): Puf; 1961.

11. Jodelet D. Folies et représentations sociales. Paris (FR): Puf; 1989. 12. Blanchet A, Gotman A. L'enquete et ses méthodes: I' entretien. Paris (FR): Nathan; 1992.

13. Vignaux G. Le discours, acteur de monde: énonciation, argumentation et cognition. Paris (FR): Ophrys; 1988.

14. Luz MT. Medicina e racionalidades médicas: estudo comparativo da medicina ocidental, contemporânea, homeopática e tradicional chinesa. In: Canesquim NA. Ciências Sociais e saúde para o ensino médico. São Paulo (SP): Hucitec; 2000. p.181-200.

15. Acúrcio FA, Guimarães MDC. Utilização de medicamentos por indivíduos HIV positivos: abordagem qualitativa. Rev Saúde Pública 1999 fevereiro; 33(1):73-84.

16. Deheinzelin D, Takagaki TY, Sartori AMC, Leite OHM, Neto VA, Carvalho CRR. Fatores preditivos de abandono de tratamento por pacientes com tuberculose. Rev Hosp Clin Fac Med São Paulo 1996 julho/agosto; 51(4):131-5.

17. Rodrigues CS. Fatores associados ao abandono do acompanhamento clínico por pacientes com infecção pelo HIV em um ambulatório público de referência. [dissertação]. Belo Horizonte (MG): Faculdade de Medicina/UFMG; 2001. onde o paciente constrói seus medos e suas esperanças" ${ }^{\text {(20) }}$. É importante ser capaz de ouvir atentamente o paciente, numa conduta que não cabe dentro de protocolos e nem se aplica a técnicas precisas e pré-estabelecidas, ou seja, uma relação impossível de ser instrumentalizada, pois com cada um será uma vivência diferente.

18. Kravitz R, Hays RD, Sherbourne CD, Di Matteo MR, Rogers WH, Ordway $L$, et al. Recall to recomendations and adherence to advice among patients with chronic medical conditions. Arch Intern Med 1993; 153:1869-78.

19. Lopes CHAF. O Significado da Nutrição parenteral para o cliente no contexto hospitalar. [dissertação]. Fortaleza (CE): Escola de Enfermagem/UFC; 1995.

20. PetitatA. La profession infirmière, um siècle de mutations. In: Aiach P, Fassin D, organizadores. La Garenne Colombes. Editions de L'Espace Européen; 1992. 